\title{
Central Odontogenic Fibroma combined with Central Giant Cell Lesion of the Mandible. Immunohistochemical profile.
}

\author{
Ronell Bologna Molina ${ }^{1}$, Laura Pacheco Ruiz ${ }^{2}$, Adalberto Mosqueda Taylor ${ }^{3}$, Héctor Gustavo Huesca Ramí- \\ rez $^{4}$, José Angel Ponce Lonato ${ }^{2}$, Rogelio González-González ${ }^{1}$ \\ ${ }^{1}$ Research Department, School of Dentistry, Juarez University of the Durango State, Durango, Mexico. \\ ${ }^{2}$ Maxillofacial Surgery Department, Centro Médico Nacional 20 de Noviembre ISSSTE, México \\ ${ }^{3}$ Health Care Department, Universidad Auónoma Metropolitana - Xochimilco. \\ ${ }^{4}$ Interdisciplinary Center for Health Sciences, Instituto Politécnico Nacional
}

\begin{abstract}
Central Odontogenic Fibroma is a benign neoplasm of mesenchymal origin that makes up less than 5\% of odontogenic tumors. There is a variation of this lesion that includes a zone of giant cells. This neoplasm is characterized by fibroblast proliferation, a component of apparently inactive odontogenic epithelium within a mature connective tissue stroma and multinucleated osteoclast-like giant cells. Clinically, it manifests as a slow-growing central lesion, which causes painless cortical expansion and may occasionally cause the displacement of adjacent dental organs or resorption. We present a new case of this combined lesion that was revealed radiographically as a well-defined radiolucent area in the left mandibular body. Histologically, we identified a mesenchymal lesion composed of mature fibrocellular tissue, where islands and cords of odontogenic epithelium and fibrocellular areas with numerous giant cells can be distinguished. The immunohistochemical examination was positive for giant cells with the marker CD68 and positive for epithelium cords with the marker CK19, which indicates that this an odontogenic lesion.
\end{abstract}

Key words: Central Odontogenic Fibroma, Central Giant Cell Lesion, CK19, CD68. 


\section{Introduction}

Central Odontogenic Fibroma (COF) is a benign neoplasm of mesenchymal origin. It is uncommon, accounting for less than $5 \%$ of odontogenic tumors. In an even lower percentage of cases, there is a variant consisting of an area of central giant cell granuloma (CGCG). This subtype is formed histologically by fibroblast proliferation and a variable component of apparently inactive odontogenic epithelium, scattered within a mature connective tissue stroma where some lesions may show osteoid deposits (1). Clinically, it manifests as a slowgrowing central lesion, which causes painless cortical expansion and may occasionally cause the displacement of adjacent dental organs or resorption (2). Radiographically, it is revealed as a unilocular or multilocular, well-defined and radiolucent area (3). It may occur in the maxilla or the mandible; when found in the maxilla, it predominates in the anterior region of the first molar, and when in the mandible, it appears more frequently in the molar region (4). The frequency of reported cases to date has shown a higher percentage of occurrence in young women (1-3). There are three pathogenic hypotheses based on the clinical and pathological characteristics. The first considers the neoplasm to be a COF with reactive CGCG component; the second proposes that it is a hybrid lesion; and the third supposes a collision of the COF/CGCG (2-4). The treatment is surgical removal of the lesion, by curettage of the surrounding area. After its removal, a well-defined but non-encapsulated specimen is obtained, which is irregular, soft and brown. It has a rough surface containing small fragments of hard tissue. Recurrences of these lesions are common (25\%) (3).

The purpose of this article is threefold. First, to report a case of a hybrid neoplasm, secondly to list the clinical, radiological and histological characteristics and thirdly to compare the results found in this neoplasm with other hybrid tumors previously reported.

\section{Case Report}

A 14-year-old male patient was afflicted with a mild volume increase in the left mandibular body that had developed over six months. The patient was asymptomatic, with no tooth displacement or resorption, firm palpation, normal characteristics in the adjacent mucosa, and a clinical history with no record of notable trauma or pathological antecedents. The panoramic radiograph revealed a well-defined unilocular radiolucent area in the body of the mandible, located below the area between the first premolar and left second molar (Fig 1a). Computed Axial Tomography (CAT) revealed a multilocular lesion with a vestibular cortical expansion (Figure 1b). An incisional biopsy was performed with a corresponding histopathological study that identified a mesenchymal lesion composed of mature fibrocellular tissue. In

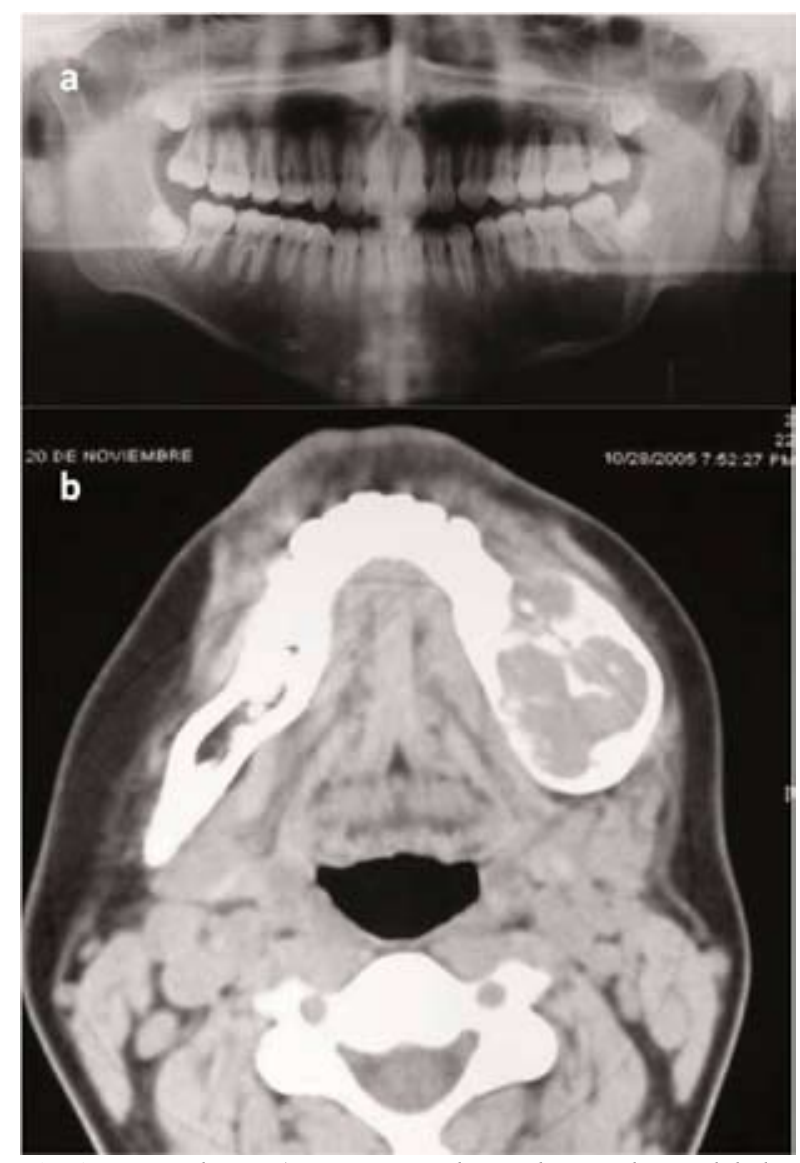

Fig. 1. Imagenology: a)Panoramic radiography reveals a unilobular lesion between the first premolar and left lower second molar, b) Computerized Axial Tomography showing a multilocular lesion with vestibular cortical expansion

this study, islands and strands of odontogenic epithelium and fibrocellular areas with numerous multinucleated giant cells can be distinguished (Fig 2a, 2b). An immunohistochemical technique was performed, which tested positive for giant cells with the marker CD68 (Fig 3a) and positive for islands and strands of odontogenic epithelium with the marker CK19 (Fig 3b), thus corroborating the combined strain of the lesion. Subsequently, we carried out a resection of the lesion and installed a Krenkel dynamic reconstruction plate. Findings: We obtained a defined but non-encapsulated specimen that was soft, brown, irregularly shaped, and had a rough surface with small fragments of hard tissue. At the surgical site, cortical expansion as well injury of the basal edge was found. Curettage was performed in the area with milling of the bone walls.

In the 2-year postoperative control, we observed facial symmetry, bone regeneration beginning at the periosteum, as well as an occlusion and alveolar ridge appropriate for future rehabilitation with dental implants, thus preserving the function and aesthetics of the patient's facial structure.

The clinical and histological characteristics of this com- 

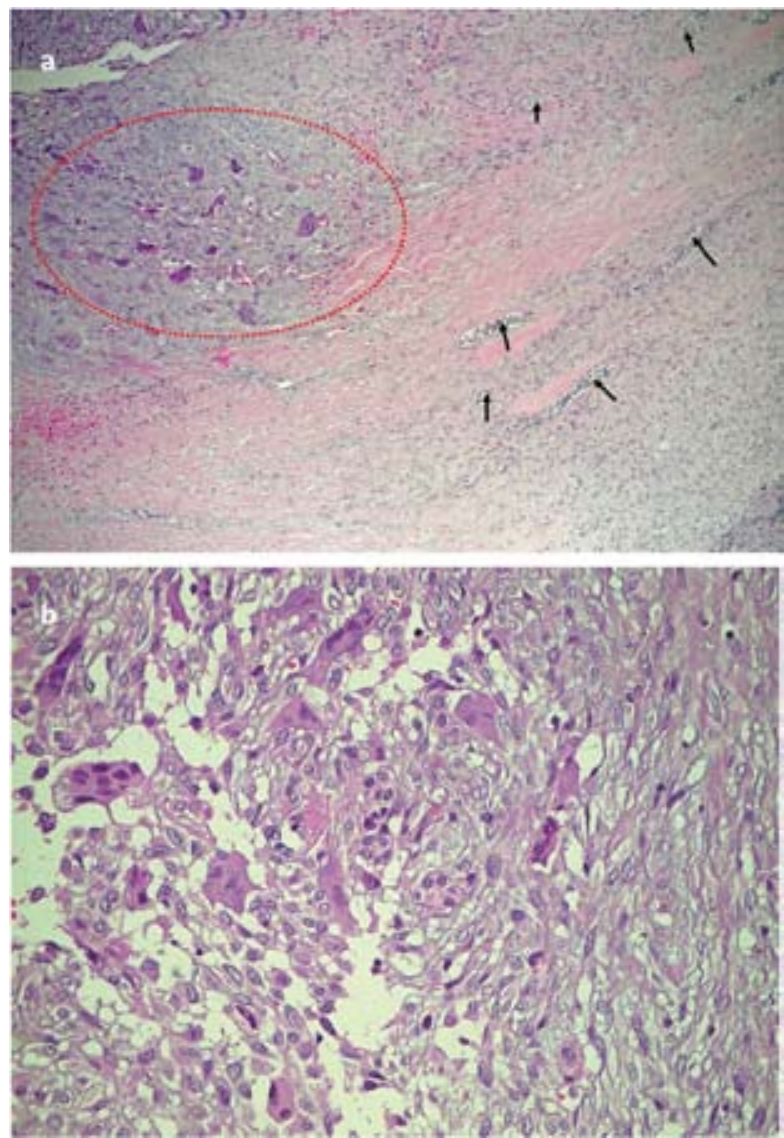

Fig. 2. Histophatology: a) Microphotography showing a giant cells (red circle) and the odontogenic epithelium (arrows), (100x).b) Incisional biopsy showing a mature fibrocellular area in which islands and cords of odontogenic epithelium are mixed with numerous multinucleated giant cells in some areas.

bination of Central Odontogenic Fibroma with Giant Cells require a detailed differential diagnosis.

The differential diagnosis that are considered to identify COF with similar lesions among them for example: mixoma, fibromixoma and a desmoplastic fibroma (4-6). Conversely, the differential diagnosis that includes giant cells lesions, can be considered cherubim, brown tumor of hyperparathyroidism and aneurismal bone cyst (7).

This components can be fully identified, which is why the indicators CK19 and CD68 were used in the immunohistochemical study.

\section{Discussion}

From 1992 until 2008 there has been 13 COF reported cases with CGCG $(4,8,9)$. Histologically these lesions present a wide range that goes from dense to delicate collagen with fibromixoid stroma and odontogenic epithelial bands that seem inactive, may seen hyaline white corpuscle, fusiform cells and intra osseous lesions mainly formed of fibro cellular tissue with multiple hemorrhagic focus, giant multinuclear cells added and in some cases immature bone formation can be observed
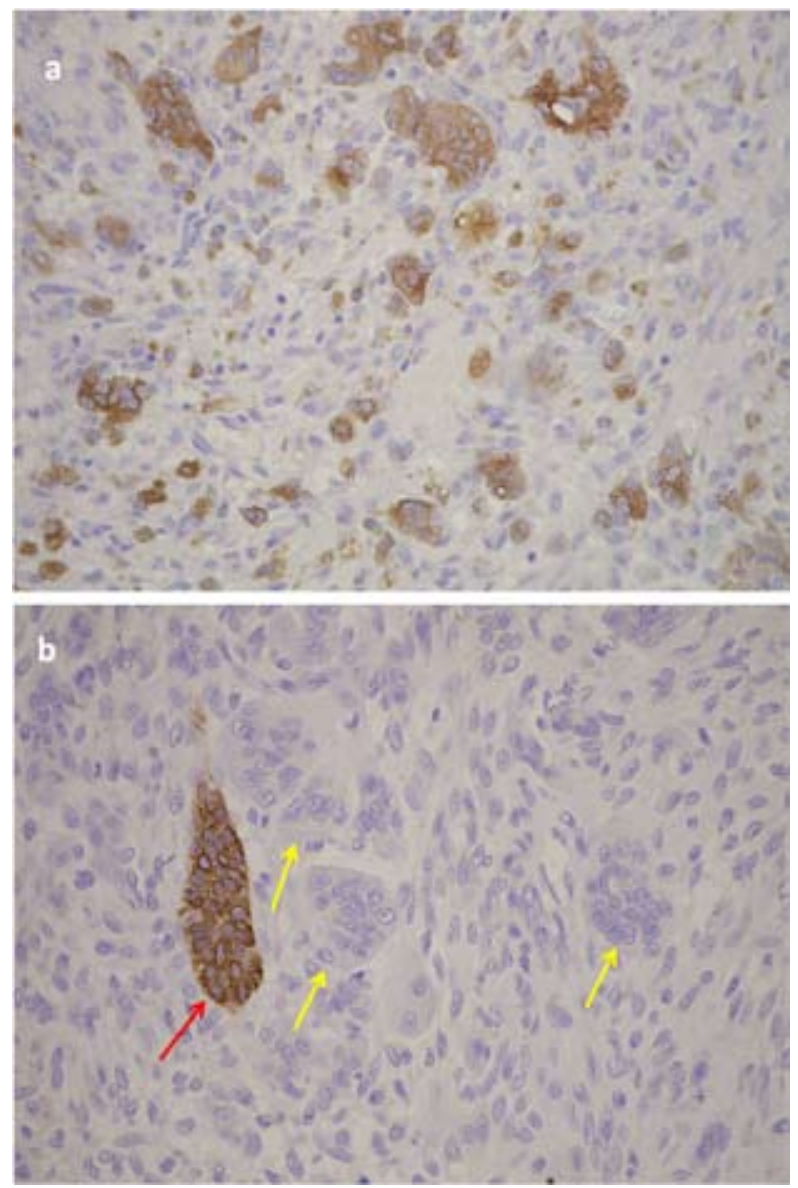

Fig. 3. Immunohistochemistry: a)Positive staining for marker CD68 in giant cells. (400X), b) Red arrow show a positive staining for marker CK19 in islands and strands of odontogenic epithelium. Yellow arrows show giant cells. (400x).

$(4,8)$.

CK19 is an intermediate filament protein responsible for the structural integrity of epithelial cells. During fetal development, it is expressed in all epithelial cells from the mesenchyme, and high positivity in the odontogenic epithelium has been reported (7). CD68 is a glycoprotein expressed on the plasma membrane of macrophages and monocytes. It is used to qualitatively identify, by optical microscopy, macrophage/monocyte lineage cells in normal and neoplastic tissues and is highly positive in multinucleated giant cells (8), hence the usefulness of using these two markers in this type of combined lesion.

The use of this immunohisotchemical markers are needed to understand these types of lesions, however the use of hematoxilin and eosin is enough to realize the histopathology diagnosis of this kind of tumors.

There are three hypothesis that intend to explain the nature of this combined lesions, the first one suggest the possibility that this lesion can be a collision tumor, this happens when two lesions are present in a synchronous way and can or cannot be mixed, or can both or just one of them be extremely small. The second hypothesis ha- 
ppens when is suggested that the $\mathrm{COF}$ generates a reactive response due to the presence of inactive odontogenic epithelium. This theory in turn suggest that a makeup giant cellular response whose cells present affinity for inactive odontogenic epithelium; histologically shows COF characteristics and giants cells present in peripheral zone. The third proposal suggest that growth factors are produced by a primary CGCG lesion that produces the proliferation of odontogenic epithelium and the formation of secondary COF posterior (4,7-9).

The cases reported so far show different proportions in the components of the lesion. Mosqueda et al.(4) and Allen et al. (8) report a greater number of cellular components for central odontogenic fibroma, including fibroblasts and epithelial areas. This fact points to the idea of an odontogenic tumor with a component of reactive giant cells. Moreover, Odell et al. (10) say that the clinical features of this lesion, such as age distribution, a higher incidence in females, a predominance in the mandibular region, and cortical expansion, make it look more like central giant cell granuloma. However, these clinical manifestations are also associated with central odontogenic fibroma when present in the mouthpiece (2); therefore, it cannot be established that this lesion stems from central giant cell granuloma.

It is noteworthy that the presence of multinucleated osteoclast-like giant cells scattered throughout the fibrocellular tissue, present in other lesions, such as cherubism, aneurismal bone cysts, brown tumors from hyperparathyroidism and some other less common bone tumors, in addition to a greater number of elements of central odontogenic fibroma in this combined neoplasia, supports the idea that the giant cell component is caused by a reaction to a stimulus that cannot yet be identified, making it necessary to consider this point in future research.

\section{References}

1. Rania H, Mark A. Hybrid Central Odontogenic Fibroma with Giant Cell Granuloma-like Component: Case Report and Review of Literature. Head Neck Pathol. 2002;2:222-6

2. Tosios KI, Gopalakrishnan R, Koutlas IG. So-called hybrid central odontogenic fibroma/central giant cell lesion of the jaws. A report on seven additional cases, including an example in a patient with cherubism, and hypotheses on the pathogenesis. Head Neck Pathol. 2008;2:333-8.

3. Kaplan I, Manor I, Yahalom R, Hirshberg A. Central giant cell granuloma associated with central ossifying fibroma of the jaws: a clinicopathologic study. Oral Surg Oral Med Oral Pathol Oral Radiol Endod. 2007;103:e35-41.

4. Kaffe I, Buchner A. Radiologic features of central odontogenic fibroma. Oral Surg Oral Med Oral Pathol. 1994; 78: 811-8.

5. Mosqueda Taylor A, Bermudez Flores V, Diaz Franco M. Combined central odontogenic fibroma and giant cell granuloma- like lesion of the mandible: report of a case and review of the literature. J Oral Maxillofac Surg. 1999;57:1258-2.

6. Brannon RB. Central odontogenic fibroma, myxoma (odontogenic myxoma, fibromyxoma), and central odontogenic granular cell tumor. Oral Maxillofac Surg Clin North Am. 2004;16:359-7

7. Ikeshima A, Utsunomiya T. Case report of intraosseous fibroma: a study on odontogenic and desmoplastic fibromas with a review of the literature. J oral Sci. 2005;47:149-57

8. Regezi JA. Odontogenic cysts, odontogenic tumors, fibroosseous, and giant cell lesions of the jaws. Mod Pathol. 2002;15:331-41

9. Allen CM, Hammond HL, Stimson PG. Central odontogenic fibroma, WHO type. A report of three cases with an unusual associated giant cell reaction. Oral Surg Oral Med Oral Pathol. 1992;73:62-6

10. Odell EW, Lombardi T, Barrett AW, Morgan PR, Speight PM. Hybrid central giant cell granuloma and central odontogenic fibromalike lesions of the jaws. Histopathology. 1997;30:165-71. 